\section{A new method of polyglycolic acid sheet placement in the stomach after endoscopic submucosal dissection}

Endoscopic submucosal dissection (ESD) is a minimally invasive treatment for early gastric cancer. Further efforts are needed to decrease the complications associated with ESD, such as bleeding and perforation. A polyglycolic acid (PGA) sheet (NEOVEIL; Gunze Co., Kyoto, Japan) has the potential to prevent post-ESD bleeding because it covers post-ESD ulcers [1]. The PGA sheet is a soft, non-woven, bioabsorbable fabric that is already used clinically in respiratory and pharyngolaryngeal surgery [2]. However, difficulties are associated with the delivery of PGA sheets to the ulcer floor by endoscopy because the sheet easily sticks to itself under wet conditions. As a result, several strips of the PGA sheet need to be sequentially arranged [3]. We report a new method for PGA sheet placement in the stomach using monofilament sutures (polydioxanone: PDS II; Ethicon US LLC, Cincinnati,
Ohio, USA). We have named it "the framing method."

- Video 1 shows the procedure described here. After ESD has been performed, we measure the diameter of the ulcer ( $\bullet$ Fig. 1), cut the sheet into an oval shape of the desired size ( Fig. 2 a), and sew the circumference of the sheet with PDS II ( $\bullet$ Fig. 2b). The suture thread functions as a "frame" and keeps the sheet flat $(\bullet$ Fig. 2 c). After soaking the sheet in fibrinogen solution, artery forceps are inserted through the instrument channel of an endoscope, the sheet is grasped by the forceps, and the endoscope is inserted into the stomach. An overtube (Sumitomo Bakelite Co., Tokyo, Japan) assists the endoscope to pass smoothly through the pharynx. The sheet is dropped into the stomach and positioned on the ulcer floor ( $\bullet$ Fig.3a). An injection of fibrinogen solution and thrombin solution (Beriplast
P combi-set; CSL Behring K.K., Tokyo, Japan) promotes the adherence of the sheet to the ulcer floor ( $\bullet$ Fig. 3 ).

We successfully covered ulcers with one piece of the sheet in two patients ( $\bullet$ Fig.4). This method is easy, quick, and covers post-ESD ulcers.

Endoscopy_UCTN_Code_TTT_1AO_2AD

\section{Competing interests: None}

\section{Ken Kumagai, Satoru Iwamoto, Naoki Esaka, Yoshinori Mizumoto}

Department of Gastroenterology, National Hospital Organization Kyoto Medical Center, Kyoto, Japan

\section{References}

1 Tsuji Y, Fujishiro M, Kodashima S et al. Polyglycolic acid sheets and fibrin glue decrease the risk of bleeding after endoscopic submucosal dissection of gastric neoplasms. Gastrointest Endosc 2014; 79: 151 - 155

2 Takeuchi J, Umeda M, Murata M et al. Application of polyglycolic acid sheet and fibrin glue spray for partial glossectomy: comparison with artificial dermis. Jpn J Oral Maxillofac Surg 2011; 57: $394-400$
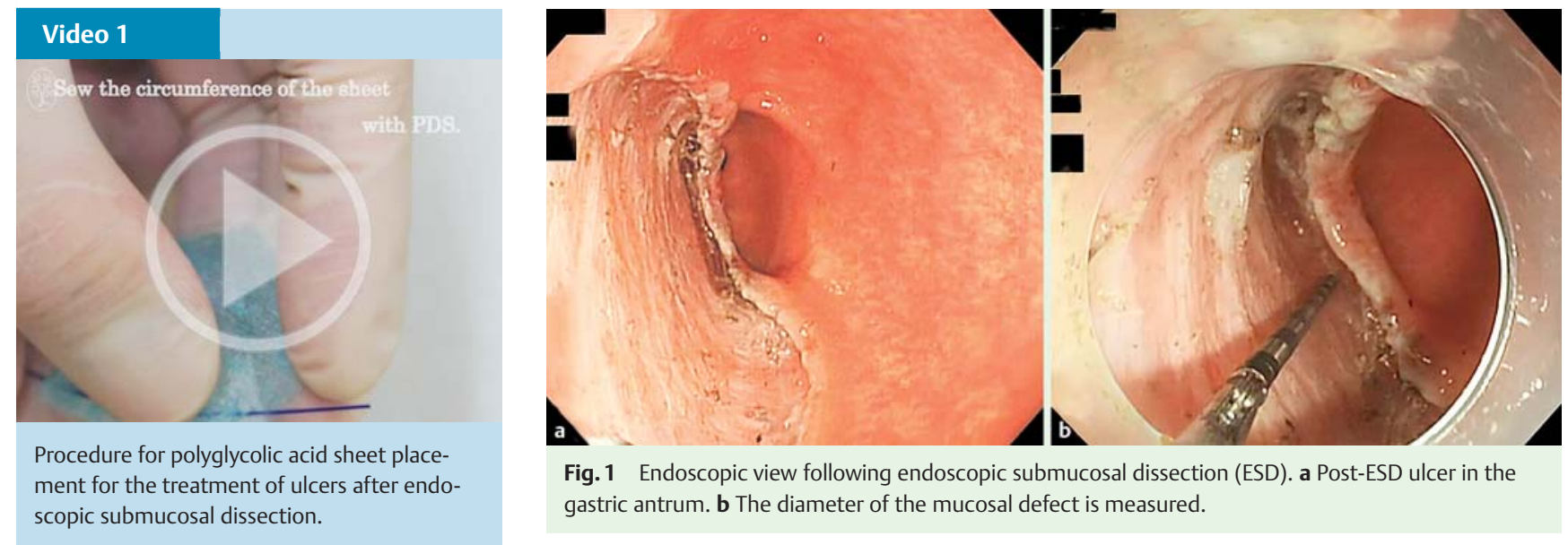

Fig. 1 Endoscopic view following endoscopic submucosal dissection (ESD). a Post-ESD ulcer in the gastric antrum. $\mathbf{b}$ The diameter of the mucosal defect is measured.
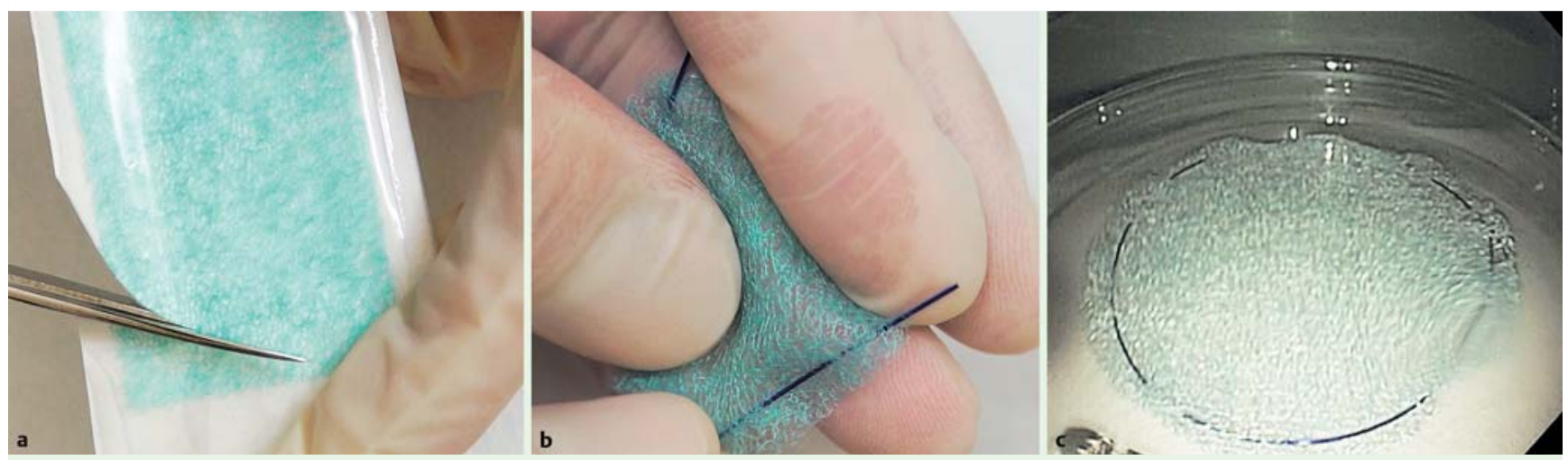

Fig. 2 Preparation of the polyglycolic acid (PGA) sheet. a The PGA sheet is cut into an oval shape of the desired size. $\mathbf{b}$ The circumference of the sheet is sewn using a polydioxanone monofilament suture thread. $\mathbf{c}$ The sheet with the circumferential thread remains flat. 


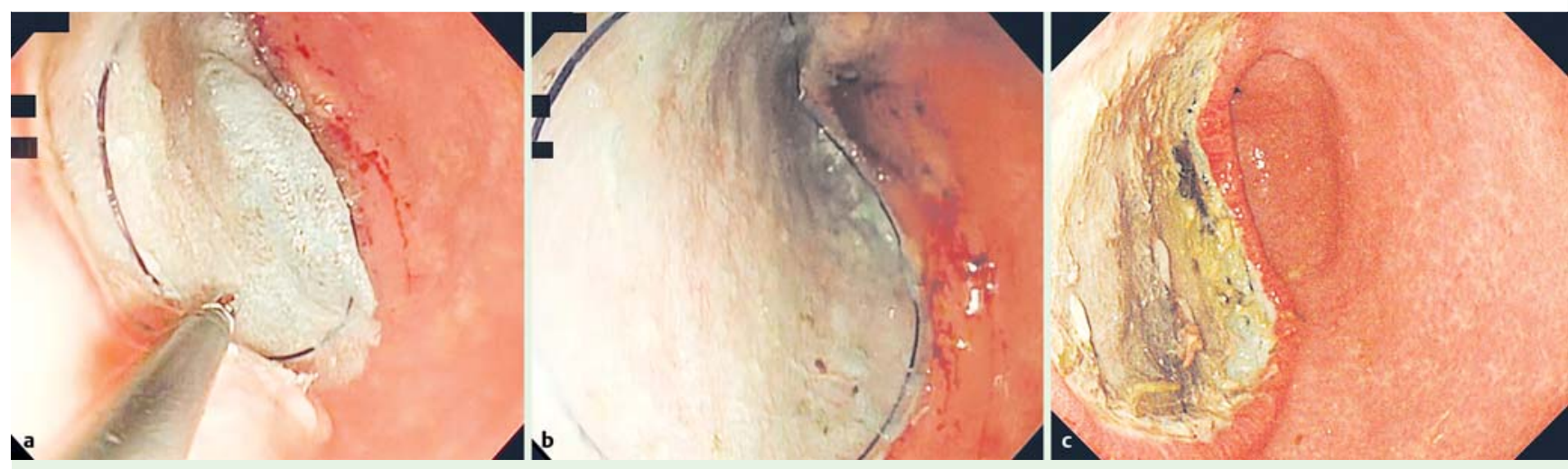

Fig. 3 Endoscopic view. a The polyglycolic acid (PGA) sheet is positioned on the ulcer floor using artery forceps. b The PGA sheet successfully covers the ulcer after placement. $\mathbf{c}$ The PGA sheet maintains its position on postoperative Day 7.

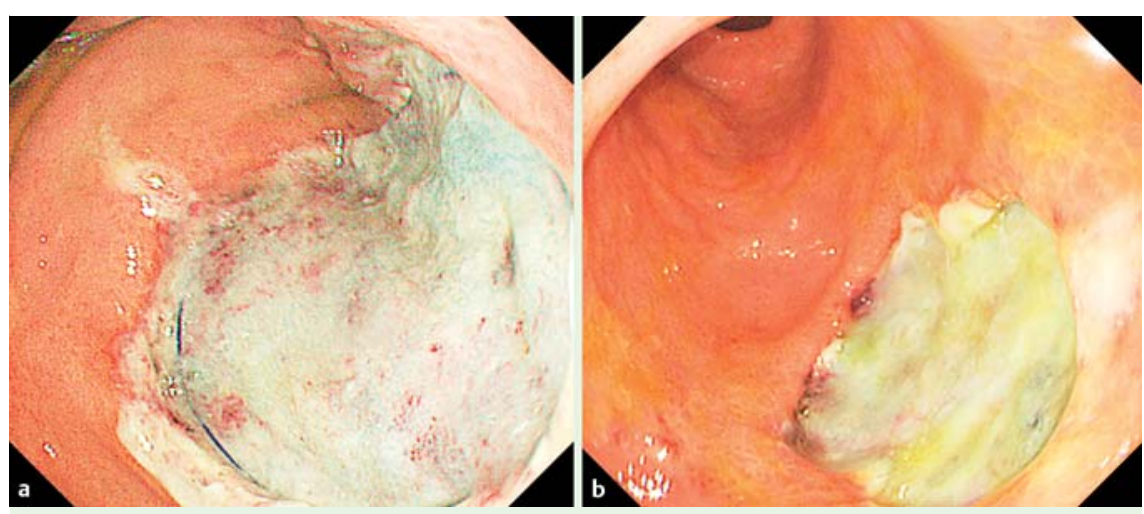

Fig. 4 Other cases of ulcer treatment following endoscopic submucosal dissection (postoperative Day 1). a Ulcer on the greater curvature of the gastric angle. $\mathbf{b}$ Ulcer on the posterior wall of the upper body of the stomach.
3 Takimoto K, Toyonaga T, Matsuyama K et al. Endoscopic tissue shielding to prevent delayed perforation associated with endoscopic submucosal dissection for duodenal neoplasms. Endoscopy 2012; 44: E414E415

\section{Bibliography}

Dol http://dx.doi.org/

10.1055/s-0042-112981

Endoscopy 2016; 48: E274-E275

(c) Georg Thieme Verlag KG

Stuttgart · New York

ISSN 0013-726X

\section{Corresponding author} Ken Kumagai, MD

Department of Gastroenterology National Hospital Organization Kyoto Medical Center

1-1, Mukaihata-cho

Fushimi-ku

Kyoto, 612-8555

Japan

Fax: +81-75-6434325

kekumaga@kyotolan.hosp.go.jp 